International Journal of Humanoid Robotics

(c) World Scientific Publishing Company

\title{
Walking Stability of a Variable Length Inverted Pendulum Controlled with Virtual Constraints
}

\author{
Qiuyue Luo* \\ Laboratoire des Sciences du Numérique de Nantes (LS2N), CNRS, Centrale Nantes, Université \\ de Nantes, 1 rue de la Nö̈ \\ Nantes, 44321, France \\ qiuyue.luo@ls2n.fr \\ Christine Chevallereau \\ Laboratoire des Sciences du Numérique de Nantes (LS2N), CNRS, Centrale Nantes, Université \\ de Nantes, 1 rue de la Nö̈ \\ Nantes, 44321, France \\ christine.chevallereau@ls2n.fr \\ Yannick Aoustin \\ Laboratoire des Sciences du Numérique de Nantes (LS2N), CNRS, Centrale Nantes, Université \\ de Nantes, 1 rue de la Nö̈ \\ Nantes, 44321, France \\ Yannick.Aoustin@univ-nantes.fr
}

Received Day Month Year

Revised Day Month Year

Accepted Day Month Year

\begin{abstract}
Bipedal walking is a complex phenomenon that is not fully understood. Simplified models make it easier to highlight important features. Here the variable length inverted pendulum (VLIP) model is used, which has the particularity of taking into account the vertical oscillations of the center of mass (CoM). When the desired walking gait is defined as virtual constraints i.e. as functions of a phasing variable and not on time, for the evolution of the swing foot and the vertical oscillation of the CoM, the walk will asymptotically converge to the periodic motion under disturbance with proper choice of the virtual constraints, thus a self-stabilization is obtained. It is shown that the vertical CoM oscillation, positions of the swing foot and the choice of the switching condition play crucial roles in stability. Moreover, a PI controller of the CoM velocity along the sagittal axis is also proposed such that the walking speed of the robot can converge to another periodic motion with a different walking speed. In this way, a natural walking gait is illustrated as well as the possibility of velocity adaptation as observed in human walking.
\end{abstract}

Keywords: Variable length inverted pendulum (VLIP); Hybrid model; Virtual constraints; Self-stabilization; Biped.

* Corresponding author. 


\section{Introduction}

Humanoid robots are very complex systems with high degrees-of-freedom (DoF). In order to study the essential factor of the walking gait, the simplified models are usually used. One of the mostly used simplified models is the 3D linear inverted pendulum (LIP) model proposed by S. Kajita ${ }^{1}$, which regards the robot as a concentrated mass with a zero vertical acceleration. The 3D LIP model is interesting because an analytical expression to define the center of mass (CoM) evolution exists and its dynamics in sagittal and frontal planes are decoupled.

However, in human walk, the height of the CoM is not constant, and the vertical oscillation of the CoM must be considered ${ }^{2 ; 3}$. Authors in Ref. ${ }^{2}$ observed that during walking the maximum vertical displacement increases from $0.013 \pm 0.001 \mathrm{~m}$ (mean \pm S.E.M.) for a walking speed $0.5 \mathrm{~ms}^{-1}$ to $0.031 \pm 0.007 \mathrm{~m}$ for a walking speed $2.5 \mathrm{~ms}^{-1}$. Several approaches have been proposed to extend the LIP to have a more human-like behavior. Omran et al. ${ }^{4}$ investigated the effect of vertical motion of the CoM during humanoid walking. Numerical tests show a consequent reduction of the robot torque solicitation when the CoM oscillates vertically. Garofalo et $a l .{ }^{5}$ mapped the simple dynamics of the bipedal spring loaded inverted pendulum (SLIP) model to multi-body robots to obtain a desired behavior for the CoM, yet an upper level controller is necessary to deal with the stabilization of the SLIP model. Harada et al. ${ }^{6}$ captured the walking motion of a human, and applied the obtained information on real humanoid robot. The variable length inverted pendulum (VLIP) model is used in this paper. Contrary to the SLIP model, a rigid model of the leg is used and the vertical oscillations of the CoM results from the control strategy. At the end of each step, the vertical velocity of the CoM points downwards, and the vertical movement of the CoM is a polynomial based on boundary conditions. It has been proven that the vertical oscillations induce a self-stabilization of the walk for specific virtual constraints ${ }^{7}$. The current paper extends these preliminary results into a more systematic study of the virtual constraint by extending the results of self-synchronization obtained with a LIP model $^{8}$.

For a robot with feet, the necessary and sufficient condition of stable locomotion is to have the Zero-Moment-Point (ZMP) within the support polygon at all stages of the locomotion gait $9 ; 10 ; 11$. For robots with point feet, this condition means at at each time, the robot is in dynamical equilibrium. In single support phase, the dynamic equilibrium defines the angular acceleration around the point of contact, and the concept of ZMP is not directly applicable to such systems. Absence of active actuation at the ankles makes these systems under-actuated. The Poincaré return map $^{12 ; 13}$ is the appropriate mathematical tool for analyzing the stability of periodic orbits for under-actuated systems. In this context, the stability is the convergence toward an orbit or periodic motion.

In order to avoid falling down, when and where to place the swing foot is essentially important. For a robot that only follows a pre-defined walking gait, a small disturbance on the ground or mass distribution may change the original walking 
direction of the robot, or even cause the robot to lose balance. In Ref. ${ }^{14}$ and Ref. ${ }^{15}$, a constant period is assumed, and a preview control over several steps is considered for gait planning. Takenaka et al. ${ }^{16}$ proposed an approach based on the divergent component of motion (DCM) to generate walking patterns with good stability properties. Hopkins et al. ${ }^{17}$ proposed a time varying DCM for better tracking of the vertical component of the DCM during walking on uneven grounds. Englsberger et al., by using the same property of decomposition into an intuitive way as two cascaded first-order systems, derived a capture point controller (CP), which exploits the natural dynamics of the LIP in order to get a gait pattern generator ${ }^{18}$. This result was extended to three dimension in Ref. ${ }^{19}$. Approaches presented in Ref. ${ }^{20}$ and Ref. ${ }^{21}$ modify both the step timing and step length on-line to control the walking speed under small or medium disturbances. However, these approaches can be only applied for the LIP model, and the stable walking is based on the prediction of future states of the CoM and foot locations.

For under-actuated robots, virtual constraints are functional relations among the system's states that are imposed via a feedback controller ${ }^{12}$. In order to synchronize the evolution of the various links, an internal phasing variable is used such as the position of robot's hip along the sagittal axis ${ }^{22}$. Griffin and Grizzle ${ }^{23}$ introduced a class of virtual nonholonomic constraints that depend on velocity through (generalized) angular momentum. Including angular momentum in the virtual constraints allows foot placement control to be designed on the basis of the full dynamic model of the biped. This method leads to a new class of control laws that are robust to a variety of common gait disturbances. Authors plane to extend this method to a 3D walking.

The objective of this paper is to find some physical conditions that lead to selfstabilization based on a simplified model with vertical CoM oscillation. A switching surface is proposed that when the robot's CoM crosses the surface, the stance foot is switched. By doing this, the step timing is not explicitly but implicitly adapted under disturbances. To make sure that the swing foot touches the ground at the same time when the CoM crosses the switching surface, the trajectory of the swing foot is defined as a function of a phasing variable based on the feedback of the robot's internal states instead of time. How the features of the switching surface and the vertical motion of the CoM affect the stability of the system has been analyzed. As an extension of our previous work ${ }^{8}$, this paper proves that for a VLIP model, self-stabilization can be obtained when the switching condition is based on a linear combination of the CoM positions along the sagittal and frontal axes. Moreover, it is proved that when the CoM velocity feedback in the sagittal plane is taken into account, the system is able to converge asymptotically to a new periodic motion without the complex procedure of finding a periodic motion.

The paper is structured as follows. In Section 2, the hybrid model of the VLIP is detailed. In Section 3, the virtual constraint and the phasing variable are introduced. In Section 4, the periodic motion of VLIP is introduced. In Section 5, the 
stability of the VLIP model with the proposed switching conditions that leads to self-stabilization of the walking gait is analyzed. Simulations illustrate the property of self-stabilization. Section 6 shows how a feedback on walking velocity can be used to adapt the periodic motion to a desired walking speed. Finally, several concluding remarks and perspectives are discussed in Section 7.

\section{Modeling}

The gait is composed of two phases: single support phase and double support phase. During the double support phase, the following hypotheses are chosen: 1) The double support phase is instantaneous; 2) The contact between the swing foot and the ground does not modify the velocity of the $\mathrm{CoM}^{24}$.

\subsection{Variable Length Inverted Pendulum (VLIP) Model}

During the single support phase, the VLIP model is used. Compared with LIP model, the VLIP model is closer to the real human walking behavior, because the height of the CoM is not constant during walking.

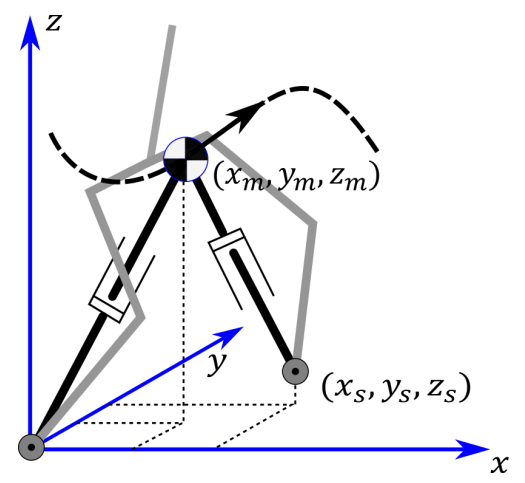

Fig. 1: The VLIP model.

The VLIP model consists of two massless legs with variable length and a concentrated mass at the hip, as shown in figure 1. The stance leg is free to rotate about the axes $x$ and $y$ at the ground. The length of each leg can be modified through actuation, allowing a desired vertical motion of the pendulum to be obtained. We also assume that the actuation of the swing leg allows a controlled displacement of the swing leg end via hip and knee actuators.

The configuration of the robot is defined via the position of the concentrated mass $\left(x_{m}, y_{m}, z_{m}\right)$ and the position of the swing foot $\left(x_{s}, y_{s}, z_{s}\right)$ with respect to a reference frame attached to the stance foot. Angular momenta along axes $x$ and 
$y$ are denoted by $\sigma_{x}$ and $\sigma_{y}$. In order to explore simultaneously the existence and stability of periodic orbits for robots with any step length and width, a dimensionless dynamic model is used ${ }^{7}$. The normalized scaling factors applied along $x$ and $y$ axes depend on desired step length $S$, desired step width $D$, and the robot mass $m$. Thus, a new set of variables is defined: $\left(X, Y, z_{m}, X_{s}, Y_{s}, z_{s}, \sigma_{X}, \sigma_{Y}\right)=$ $\left(\frac{x_{m}}{S}, \frac{y_{m}}{D}, z_{m}, \frac{x_{s}}{S}, \frac{y_{s}}{D}, z_{s}, \frac{\sigma_{x}}{m D}, \frac{\sigma_{y}}{m S}\right)$

The variables $\left(z_{m}, X_{s}, Y_{s}, z_{s}\right)$ are chosen to be the controlled variables determined by virtual constraints as functions of the phasing variable $\Phi(X, Y)$, where $X$ and $Y$ are defined as free variables or uncontrolled variables $q_{f}=(X, Y)$. The definition of the phasing variable will be discussed in detail in Section 3.2. The height of the CoM and its velocity can be expressed as:

$$
\begin{aligned}
& z_{m}=f(\Phi) \\
& \dot{z}_{m}=\frac{\partial f(\Phi)}{\partial \Phi}\left(\frac{\partial \Phi}{\partial X} \dot{X}+\frac{\partial \Phi}{\partial Y} \dot{Y}\right)=\frac{\partial f}{\partial X} \dot{X}+\frac{\partial f}{\partial Y} \dot{Y}
\end{aligned}
$$

Since the legs are massless, the motion of the swing leg does not affect the dynamic model. The moment balance equation of the pendulum around the rotation axes $x$ and $y$, with the normalized variable, directly yields the dynamic equation:

$$
\left\{\begin{array}{l}
\dot{\sigma}_{X}=-g Y \\
\dot{\sigma}_{Y}=g X
\end{array}\right.
$$

For the VLIP, the angular momentum is:

$$
\left\{\begin{array}{l}
\sigma_{X}=\dot{z}_{m} Y-z_{m} \dot{Y} \\
\sigma_{Y}=-\dot{z}_{m} X+z_{m} \dot{X}
\end{array}\right.
$$

Assuming that the control law allows that the vertical motion of the CoM is perfectly tracked, Eq. (1) can be used to rewrite the zero dynamics ${ }^{12}$ as :

$$
\left\{\begin{array}{l}
\dot{q}_{f}=M_{X Y}^{-1} \cdot\left[\sigma_{X} ; \sigma_{Y}\right]^{\top} \\
\dot{\sigma}_{X}=-g Y \\
\dot{\sigma}_{Y}=g X
\end{array}\right.
$$

where

$$
M_{X Y}=\left[\begin{array}{rr}
\frac{\partial f}{\partial X} Y & \frac{\partial f}{\partial Y} Y-f \\
f-\frac{\partial f}{\partial Y} Y & -\frac{\partial f}{\partial Y} X
\end{array}\right]
$$

\subsection{Transition between steps}

Due to the hypothesis of massless legs, the contact between the swing foot and the ground does not affect the velocity of the CoM, and the velocity of CoM is conserved at each transition of stance leg. Since the reference frame is always attached to the stance foot and the $y$ axis is directed toward the CoM, the sign of the velocity along 
$y$ axis will be changed from positive to negative, i.e.

$$
\begin{aligned}
& \dot{X}_{k+1}^{+}=\dot{X}_{k}^{-}, \\
& \dot{Y}_{k+1}^{+}=-\dot{Y}_{k}^{-}, \\
& \dot{z}_{m, k+1}^{+}=\dot{z}_{m, k}^{-}
\end{aligned}
$$

The state before the transition, i.e. at the end of a step, is expressed by superscript $^{-}$and that after the transition, i.e. at the beginning of a step, is expressed by ${ }^{+}$. The variables corresponding to the step $k$, are denoted with index $k$, while those of the next step are denoted with $k+1$.

After transition, the swing foot placement becomes the new stance foot placement. Thus the CoM position after transition along $x$ axis equals to the CoM position before transition minus the swing foot position. Similar result can be obtained for the CoM position along $y$ axis:

$$
\begin{aligned}
& X_{k+1}^{+}=X_{k}^{-}-X_{s, k}^{-} \\
& Y_{k+1}^{+}=-Y_{k}^{-}+Y_{s, k}^{-}
\end{aligned}
$$

Knowing the final state of the single support phase, the transition model (6) and (7) determines the initial state of the ensuing single support phase.

\subsection{Hybrid model.}

The transition occurs when the height of the swing foot $z_{s}$ is zero. A switching manifold is defined below:

$$
\mathscr{S}:=\left\{\mathbf{x} \mid z_{s}=0, \dot{z}_{s}<0\right\}
$$

where $\mathbf{x}:=\left[X, Y, z_{m}, X_{s}, Y_{s}, z_{s}, \dot{X}, \dot{Y}, \dot{z}_{m}, \dot{X}_{s}, \dot{Y}_{s}, \dot{z}_{s}\right]^{\top}$ is the state of the robot. The transition model (6) and (7) can be rewritten as:

$$
\mathbf{x}^{+}=\Delta\left(\mathbf{x}^{-}\right)
$$

where $\Delta$ indicates the transition map. Thus the combination of the dynamic equations and the transition model (9) forms the single-phase hybrid dynamical system:

$$
\Sigma: \begin{cases}\dot{\mathbf{x}}=f(\mathbf{x})+g(\mathbf{x}) u, & \mathbf{x}^{-} \notin \mathscr{S} \\ \mathbf{x}^{+}=\Delta\left(\mathbf{x}^{-}\right), & \mathbf{x}^{-} \in \mathscr{S}\end{cases}
$$

where $u$ is the control law that allows us to track the reference trajectory of the swing foot and vertical motion of the CoM.

\section{Virtual Constraints}

A normalized variable $\Phi$ monotonically increasing from 0 to 1 during one step, named phasing variable is defined to describe the desired trajectories of the controlled variables. The trajectories of the swing foot and the vertical oscillation of CoM are defined as a function of $\Phi: X_{s}=X_{s}(\Phi), Y_{s}=Y_{s}(\Phi), z_{s}=z_{s}(\Phi)$, $z_{m}=z_{m}(\Phi)$. The vertical evolution of swing foot determines the step timing while 
the horizontal evolution determines foot locations. The advantage of introducing virtual constraints based on a phasing variable is that this method can be extended to a complete model of robots by using the phasing variable to coordinate all the joint motions of the robot.

\subsection{Switching manifold}

In paper ${ }^{8}$, it has been proven that when the transition of stance foot is based on time, the state of the robot will not converge to the fixed value when there exists an initial perturbation. Instead of time, the phasing variable based on the CoM position $X$ and $Y$ proposed in paper $^{8}$ is used here.

The vertical trajectory of the swing foot is defined as a function of $\Phi(X, Y)$. The transition happens when the swing foot touches the ground, which defines a relationship between the two variables $X$ and $Y$. An infinite number of CoM positions satisfy it. This set of positions are grouped in the switching configuration manifold defined by:

$$
\mathbb{S}=\left\{(X, Y) \mid z_{s}(\Phi)=0\right\}
$$

The phasing variable is chosen such that the robot switches its stance leg when the CoM crosses the switching manifold:

$$
\mathbf{S}=\left\{(X, Y) \mid\left(X-X^{*-}\right)+C\left(Y-Y^{*-}\right)=0\right\} .
$$

The switching manifold $\mathbf{S}$ is defined as a vertical surface parameterized by $C$, represented by the gray surface in figure 2. Many other sets of positions can be considered but since stability studied here is a local property, a flat surface is a convenient choice. The choice of $\mathbf{S}$ directly affects the final CoM position for step $k$.

\subsection{Phasing Variable}

As introduced in paper $^{8}$, the phasing variable $\Phi$ is defined as a function of $X$ and $Y$ with six parameters:

$$
\Phi=a X+b Y+c X Y+d X^{2}+e Y^{2}+f
$$

and the phasing variable must vary from 0 to 1 for a step and be monotonic. Since the phasing variable must be zero at the beginning of a step, $\Phi\left(X^{+}, Y^{+}\right)=0$, where $X^{+}$and $Y^{+}$are known from Eq. (7). Besides, in order to make the switching manifold (11) and (12) equivalent, the phasing variable at the end of a step $\Phi\left(X^{-}, Y^{-}\right)=1$, for any $X^{-}=X^{*-}-C \delta$ and $Y^{-}=Y^{*-}+\delta$, where $\delta$ is the CoM position error along $y$ axis at the end of a step. Since all the above constraints must be satisfied for an arbitrary value of $\delta$, three more equations can be obtained and 


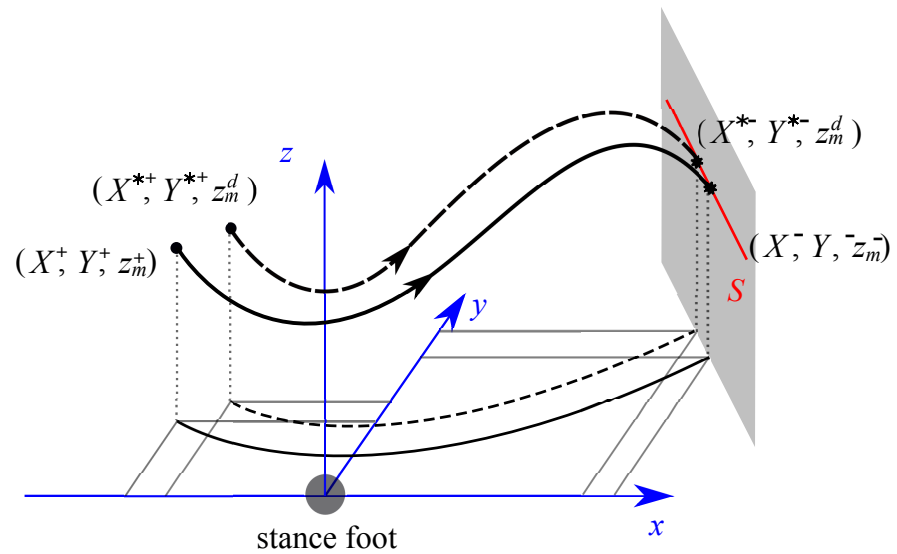

Fig. 2: The step finishes when the CoM crosses the switching manifold, which is represented by the gray surface. The curved dashed line is the periodic motion, and the curved solid line the CoM motion under an initial perturbation.

we have:

$$
\left\{\begin{aligned}
a X^{+}+b Y^{+}+c X^{+} Y^{+}+d\left(X^{+}\right)^{2}+e\left(Y^{+}\right)^{2}+f & =0 \\
a X^{*-}+b Y^{*-}+c X^{*-} Y^{*-}+d\left(X^{*-}\right)^{2}+e\left(Y^{*-}\right)^{2}+f & =1 \\
-c C+d C^{2}+e & =0 \\
-a C+b-c C Y^{*-}+c X^{*-}-2 d C X^{*-}+2 e Y^{*-} & =0 .
\end{aligned}\right.
$$

There are six variables $\{a, b, c, d, e, f\}$ while the number of equations is only four, thus parameters $a, b, e$, and $f$ can be obtained as functions of $c$ and $d$. The values of $c$ and $d$ can be chosen based on some optimization standards. Here, the objective function is chosen to minimize the difference between $\Phi(t)$ and $t$ to insure the monotony of $\Phi$. That is:

$$
J=\Sigma\left(\Phi(t)_{i}-t_{i}\right)^{2}
$$

The matlab function fmincon $\AA$ is used off-line when virtual constraint are designed.

\subsection{The Vertical Motion of the swing foot}

An intermediate value of the phasing variable $0<\Phi_{m}<1$ is defined, so that when $\Phi=\Phi_{m}, h_{z}=\max _{0<\Phi<1}\left\{z_{s}\right\}$. For the vertical motion of swing foot, the boundary conditions are:

$$
\begin{aligned}
& z_{s}(0)=0, \quad z_{s}\left(\Phi_{m}\right)=h_{z}, \quad z_{s}(1)=0, \\
& \dot{z}_{s}(0)=0, \quad \dot{z}_{s}\left(\Phi_{m}\right)=0, \quad \dot{z}_{s}(1)=v_{z},
\end{aligned}
$$


where $h_{z}$ is the desired height of the swing foot when $\Phi=\Phi_{m}$, and $v_{z}<0$ denotes the desired downward velocity of the swing foot at the end of a step. In this paper, $z_{s}(\Phi)$ is defined as a cubic spline.

\subsection{The Horizontal Motion of the swing foot}

The position where to land the swing foot must be chosen cautiously. In order to analyze several cases, the swing foot positions at the end of step $k$ is expressed in a generalized form:

$$
\begin{aligned}
& X_{s, k}^{-}=\left(1-k_{S}\right)\left(X_{k}^{-}-X^{*+}\right)+k_{S} S \\
& Y_{s, k}^{-}=\left(1-k_{D}\right)\left(Y_{k}^{-}+Y^{*+}\right)+k_{D} D
\end{aligned}
$$

where $0 \leq k_{S} \leq 1$ and $0 \leq k_{D} \leq 1$. How the values of $k_{S}$ and $k_{D}$ affect the swing
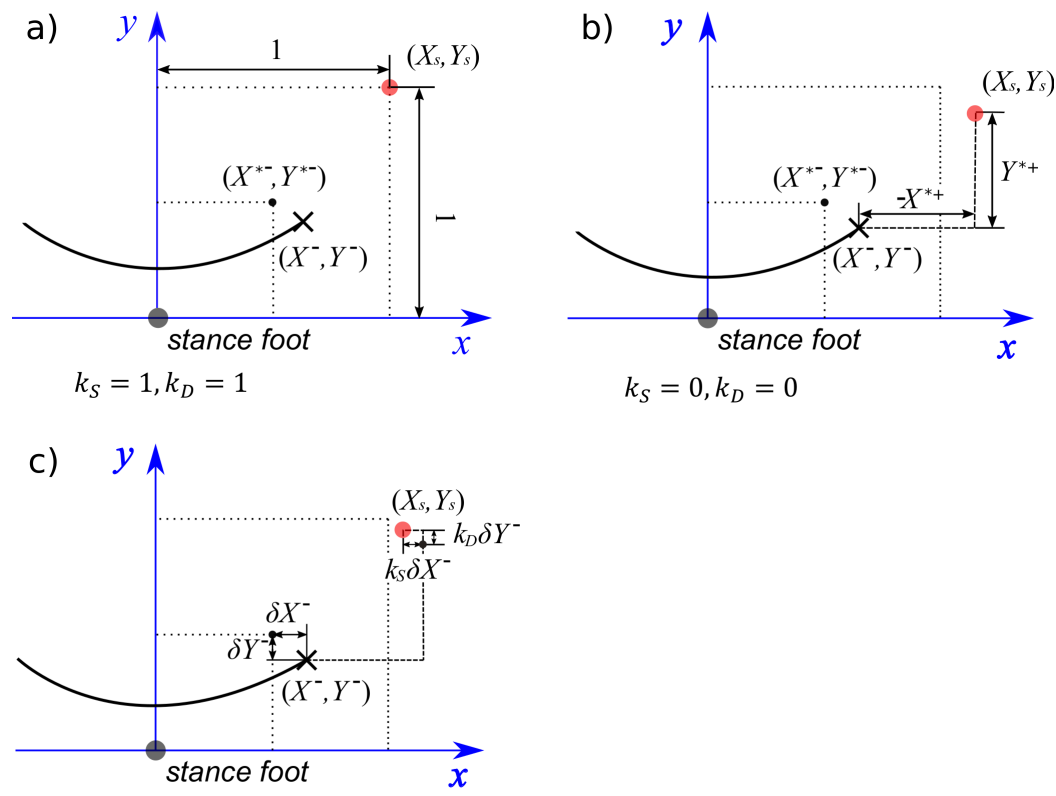

General Case

Fig. 3: Influence of $k_{S}$ and $k_{D}$ on the foot locations. a) Step length and width are fixed; b) The initial CoM position error is nullified; c) The general case. The black and the red dots represent respectively the stance feet during the current and the next steps. The curved line represents the CoM trajectory, and the cross the CoM position at the end of the current step.

foot positions is illustrated in figure 3 . The case $k_{S}=k_{D}=0$ allows to nullify the CoM position error at the beginning of the next step, i.e. $\delta X_{k+1}^{+}=X_{k+1}^{+}-X^{*+}=$ 
$0, \delta Y_{k+1}^{+}=Y_{k+1}^{+}-Y^{*+}=0$. The case $k_{S}=k_{D}=1$ corresponds to fixed step length and width.

The boundary conditions for the motion of the swing foot along $x$ direction $X_{s}(\Phi)$ are:

$$
\begin{array}{ll}
X_{s}(0)=X_{s, k}^{+}, & \dot{X}_{s}(0)=0, \\
X_{s}(1)=X_{s, k}^{-}, & \dot{X}_{s}(1)=0,
\end{array}
$$

where $X_{s, k}^{+}$is the swing foot position at the beginning of step $k$, and it can be known according to the information of the previous step. The velocity at the end of the step is chosen null to have a vertical motion of the swing foot and to increase robustness with respect ground disturbance.

The same constraints are imposed on the motion along $y$ direction $Y_{s}(\Phi)$. With these boundary conditions, the trajectories along $x$ and $y$ directions can be defined as third order polynomial functions of $\Phi$.

\subsection{Vertical Oscillation of the CoM}

It has been proven in Ref. ${ }^{7}$ that the vertical oscillation of the CoM can asymptotically stabilize a periodic walking gait. A polynomial that keeps the motion of CoM continuous is used considering the following constraints:

$$
\begin{gathered}
z_{m}(0)=z_{m}^{+} ; z_{m}(1)=z_{m}^{d} ; \\
\dot{z}_{m}(0)=\dot{z}_{m}^{+} ; \dot{z}_{m}(1)=\dot{z}_{m}^{d}
\end{gathered}
$$

The oscillations chosen are such that the final velocity for each step is negative i.e. $\dot{z}_{m}^{d}<0$.

\section{Periodic gait of VLIP}

For a VLIP, the change of support is characterized by a change of the point where the angular momentum is calculated, thus we have ${ }^{7}$ :

$$
\left\{\begin{array}{l}
\sigma_{X}^{+}=\sigma_{X}^{-}-D \dot{z}_{m}^{-} \\
\sigma_{Y}^{+}=-\sigma_{Y}^{-}+D \dot{z}_{m}^{-}
\end{array}\right.
$$

As the vertical velocity of the CoM right before the change of support is negative, it implies that the angular momenta $\sigma_{X}$ and $\sigma_{Y}$ decrease at the change of support. In order to obtain a periodic motion, the angular momenta must therefore increase during the stance phase, and thus it is necessary to slightly shift the relative position of the support leg and the CoM. The position of the CoM at the beginning of the step is then written as $X^{+}=-1 / 2+D_{S}, Y^{+}=1 / 2-D_{D}$ and at the end of the step $X^{-}=1 / 2+D_{S}, Y^{-}=1 / 2+D_{D}$ with $D_{S}=\frac{X^{+}+X^{-}}{2} \geq 0$ and $D_{D}=\frac{Y^{-}-Y^{+}}{2}$, as shown in figure 4 .

Since the model of walking is a hybrid model as shown in Section 2.3, a classical tool to defined a periodic motion is to define the fixed point of the Poincaré return 


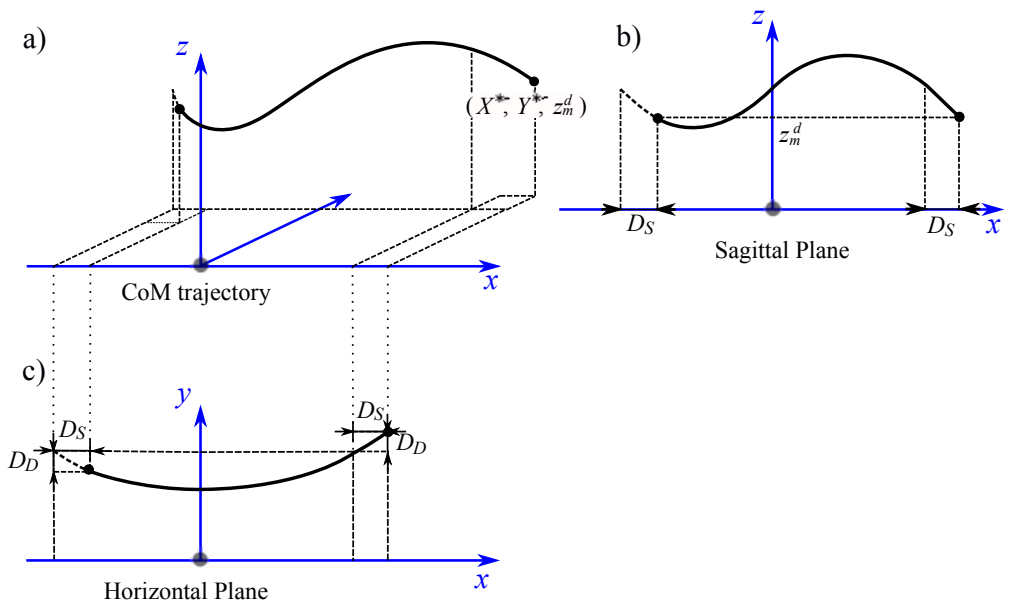

Fig. 4: The CoM trajectory of a periodic motion, and its projections in sagittal and horizontal planes.

map. In this study, we assume that a perfect tracking of the controlled variables is obtained, and the Poincaré section is defined by Eq. (12). In this context, since virtual constraints expressed as functions of $\Phi(X, Y)$ are used, the state of the robot in the Poincaré section is reduced to $\left[X^{-} ; Y^{-} ; \dot{X}^{-} ; \dot{Y}^{-}\right]$and the Poincaré return map can be written: .

$$
\left[X_{k+1}^{-}, Y_{k+1}^{-}, \dot{X}_{k+1}^{-}, \dot{Y}_{k+1}^{-}\right]=f\left(X_{k}^{-}, Y_{k}^{-}, \dot{X}_{k}^{-}, \dot{Y}_{k}^{-}\right)
$$

The periodic motion is characterized by the fixed point of the Poincaré return map and satisfy :

$$
\left[X_{k}^{-}, Y_{k}^{-}, \dot{X}_{k}^{-}, \dot{Y}_{k}^{-}\right]-f\left(X_{k}^{-}, Y_{k}^{-}, \dot{X}_{k}^{-}, \dot{Y}_{k}^{-}\right)=0
$$

The elements $D_{S}=X_{k}^{-}-1 / 2, D_{D}=Y_{k}^{-}-1 / 2, \dot{X}_{k}^{-}, \dot{Y}_{k}^{-}$are calculated by a given initial guess and matlab function $f$ solve $($ ) with Eq. $(22)$. As this optimization technique is applied for normalized parameters, the corresponding periodic steps for any step length and step width can be deduced.

\section{The stability of control strategy applied to the VLIP model}

The stability of the walking gait based on the virtual constraints defined in Section 3 is now analyzed via the stability of the Poincaré return map. A stable walking is obtained when all the norms of the eigenvalues of the Jacobian of the poincaré return map are strictly less than 1 .

Since $X^{-}$and $Y^{-}$are coupled via the switching manifold, the Jacobian matrix of the Poincaré return map at the fixed point and its eigenvalues are calculated numerically in the coordinate system $[X, \dot{X}, \dot{Y}]$. 


\subsection{Influence of $k_{S}$ and $k_{D}$ on the stability}

The values of $k_{S}$ and $k_{D}$ determine the landing position of the swing foot, and consequently the initial CoM position for the next step. From the study with the LIP model $^{8}$, we have obversed that theses parameters are crucial for the stability of walking, thus the effect of these parameters is now considered in the case of VLIP model. Cases for different values of $k_{S}$ and $k_{D}$ are studied here. The other parameters are: $\dot{z}_{m}^{d}=-0.1 \mathrm{~m} / \mathrm{s}, z_{m}^{d}=0.7 \mathrm{~m}$. The value $\dot{z}_{m}^{d}=-0.1 \mathrm{~m} / \mathrm{s}$ has been chosen according to the human vertical oscillations of the CoM. It has to be noted that in our study the double support phase is considered as an instantaneous phase, while it is not instantaneous in human walk. This assumption of instantaneous phase is made to simplify the study and due to the fact that it increases the difficulty to have stable walking as the stability margin can be improved using double support phase ${ }^{25}$. The evaluation of a pertinent choice of $\dot{z}_{m}^{d}$ to mimic human like gait is not obvious since it varies with the placement of the instantaneous double support phase in the gait, thus we consider that a value $0 \leq \dot{z}_{m}^{d} \leq-0.2 \mathrm{~m} / \mathrm{s}$ is convenient.

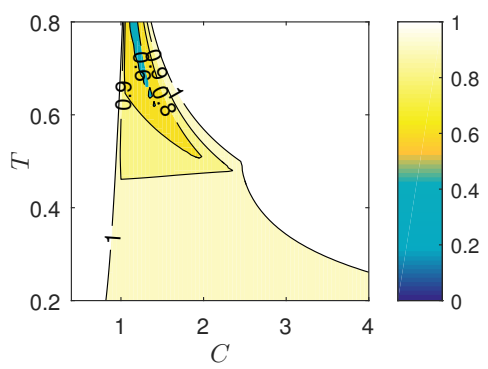

(a) $k_{S}=k_{D}=0$

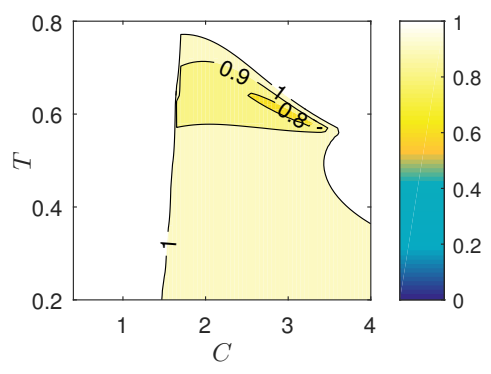

(c) $k_{S}=0, k_{D}=1$

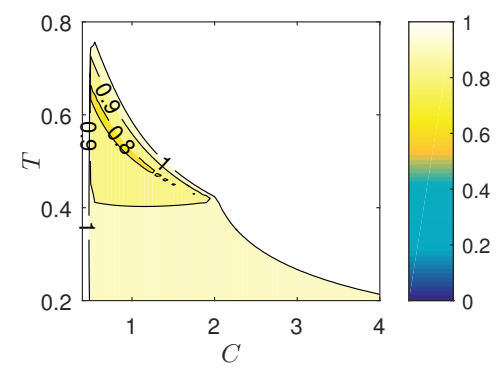

(b) $k_{S}=1, k_{D}=0$

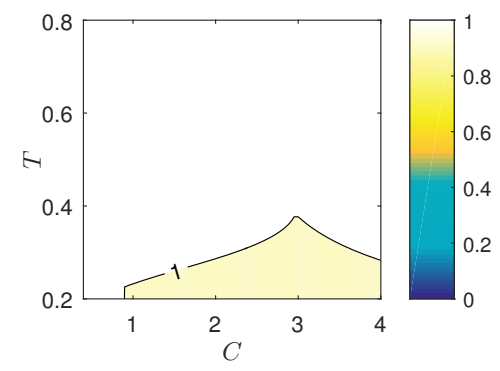

(d) $k_{S}=k_{D}=1$

Fig. 5: Influence of $k_{S}$ and $k_{D}$ on the eigenvalues. Contrary to the white areas, the colored areas indicate self-stabilization condition.

From figure 5 , it can be seen that when $k_{S}$ and $k_{D}$ are smaller, that is when the 
initial CoM error in position is smaller, larger stability region can be obtained. When $k_{S}=1$, the proper value of $T$ for stability is too small, only very fast step can be achieved with stability. Thus $k_{S}=0$ appears to be a good choice for stable walking. The choice of $k_{D} \leq 1$ has a smaller effect on the possibility to have stable walk with sufficient step duration. In the following we will consider the case $k_{S}=0, k_{D}=0$, that corresponds to placing the foot in order to nullify the initial error on the CoM position in horizontal plane for the next step. In presence of perturbation, this choice will induce some deviation in the lateral axis since $k_{D}=0$ will prevent the robot to have a strict straight line motion ${ }^{7}$. In order to be able to avoid this lateral motion, the specific case $k_{S}=0, k_{D}=1$ is also considered for the following sections where the influence of the choice of $\dot{z}_{m}^{d}$ is considered.

\subsection{Influence of $C, T$ and $\dot{z}_{m}^{d}$ on the stability}

In order to study the influence of $C, T$ and $\dot{z}_{m}^{d}$ on the eigenvalues, the values of $z_{m}^{d}$ is taken as constant: $z_{m}^{d}=0.7 \mathrm{~m}$. The results of the eigenvalues as a function of $C$ and $T$ for $\dot{z}_{m}^{d}=-0.001 \mathrm{~m} / \mathrm{s}, \dot{z}_{m}^{d}=-0.01 \mathrm{~m} / \mathrm{s}, \dot{z}_{m}^{d}=-0.1 \mathrm{~m} / \mathrm{s}$ and $\dot{z}_{m}^{d}=-0.2 \mathrm{~m} / \mathrm{s}$ are calculated for the cases $k_{S}=0, k_{D}=0$ (shown in figure 6) and $k_{S}=0, k_{D}=1$ (shown in figure 7).

It can be seen from figures 6 and 7 that the bigger $\dot{z}_{m}^{d}$ is, the smaller the eigenvalue is, which means faster convergence. For the case $k_{S}=0, k_{D}=1$, along with the increase of $\dot{z}_{m}^{d}$, the proper values of $T$ and $C$ that satisfy the stability condition will decrease. When $k_{S}=0, k_{D}=0$, the eigenvalues are smaller than the case $k_{S}=0, k_{D}=1$, but the safety margin is smaller when $T$ is big. In order to obtain self-stabilization, the value of $\dot{z}_{m}^{d}$ must be chosen cautiously according to the desired step timing or walking velocity.

\subsection{Simulations}

In order to test the robustness and self-stabilization of the walking algorithm, simulations for $k_{S}=0, k_{D}=0$ and $k_{S}=0, k_{D}=1$ are made. The step length and width $S=0.3 \mathrm{~m}, D=0.15 \mathrm{~m}$ are taken, while the desired height and vertical velocity of the CoM at the end of a step for a periodic motion are: $z_{m}^{d}=0.7 \mathrm{~m}$ and $\dot{z}_{m}^{d}=-0.1 \mathrm{~m} / \mathrm{s}$ respectively. When $T=0.6 \mathrm{~s}$, according to the results shown in figures $6 \mathrm{c}$ and $7 \mathrm{c}$, $C=1.2$ and $C=2.5$ are proper values for obtaining self-stabilization when $k_{S}=0$, $k_{D}=0$ and $k_{S}=0, k_{D}=1$ respectively. When the key parameters describing the walking are chosen, the virtual constraints can be defined (see Section 3) and the periodic motion can be calculated (see Section 4).

In the simulations, we integrate a starting and stopping configuration where the robot is assumed to be in double support and to have a rest configuration. For the first step, the CoM of VLIP starts from a position close to the future stance foot with a zero velocity, and for the last step, the CoM stops at a position close to the previous stance foot. As for the first and the last steps, the motion of the CoM is far from the periodic motion, the phasing variable is chosen to be a function of 


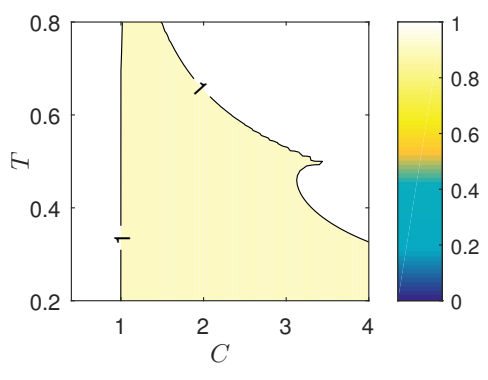

(a) $\dot{z}_{m}^{d}=-0.001 \mathrm{~m} / \mathrm{s}$

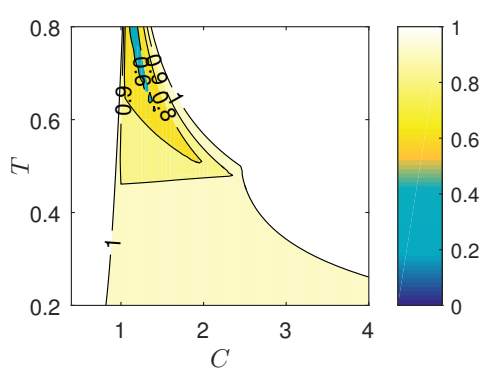

(c) $\dot{z}_{m}^{d}=-0.1 \mathrm{~m} / \mathrm{s}$

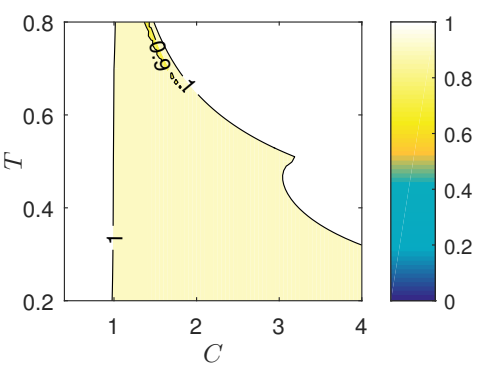

(b) $\dot{z}_{m}^{d}=-0.01 \mathrm{~m} / \mathrm{s}$

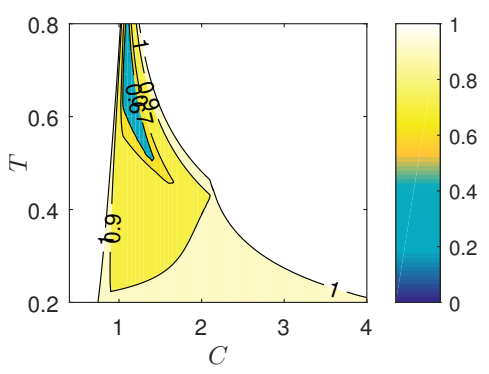

(d) $\dot{z}_{m}^{d}=-0.20 \mathrm{~m} / \mathrm{s}$

Fig. 6: Maximal norm of eigenvalues for different $\dot{z}_{m}^{d}$ when $k_{S}=0, k_{D}=0$. Contrary to the white areas, the colored areas indicate self-stabilization condition.

time. In order to obtain a CoM velocity of the VLIP in horizontal plane close to the periodic one at the end of the first step, the LIP model is used to estimate the initial CoM position for the first step $^{24}$ :

$$
\begin{aligned}
x_{0} & =\frac{\dot{x}^{*}}{\sinh (\omega T) \omega} ; \\
y_{0} & =\frac{\dot{y}^{*}}{\sinh (\omega T) \omega}
\end{aligned}
$$

where $\omega=\sqrt{g / z_{m}}$ characterizes the LIP. With the same initial CoM position, at the end of the first step, the CoM of the VLIP will end up with smaller velocity along $x$ axis than LIP. In order to increase the safety margin, the maximum CoM height of the VLIP is taken when calculating $x_{0}$ and $y_{0}$. At the last step, an optimization method is applied so that the CoM will stop to rest. 


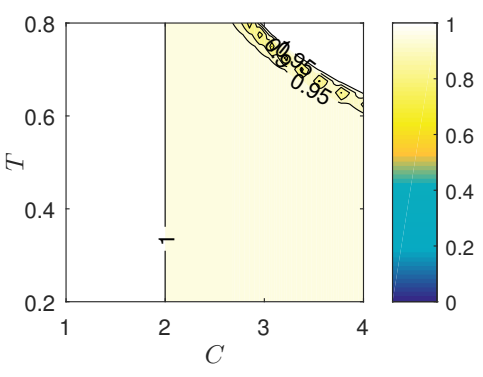

(a) $\dot{z}_{m}^{d}=-0.001 \mathrm{~m} / \mathrm{s}$

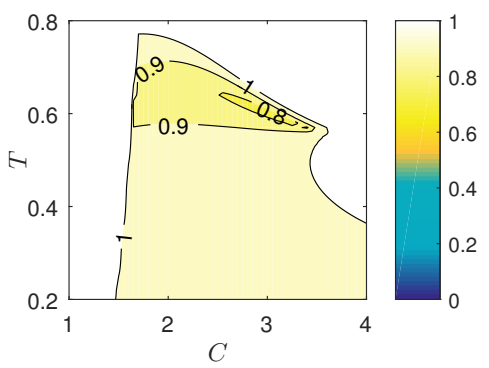

(c) $\dot{z}_{m}^{d}=-0.1 \mathrm{~m} / \mathrm{s}$

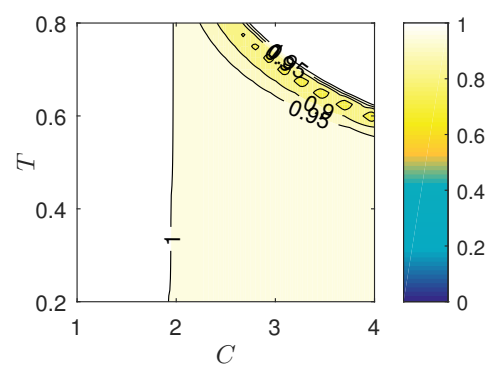

(b) $\dot{z}_{m}^{d}=-0.01 \mathrm{~m} / \mathrm{s}$

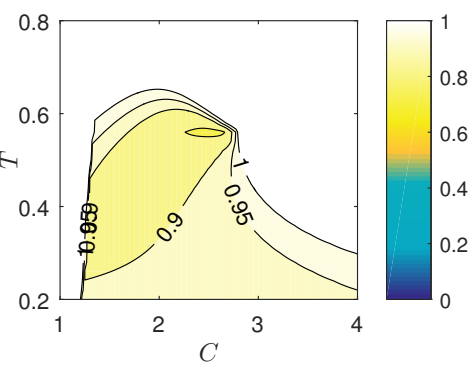

(d) $\dot{z}_{m}^{d}=-0.20 \mathrm{~m} / \mathrm{s}$

Fig. 7: Maximal norm of eigenvalues for different $\dot{z}_{m}^{d}$ when $k_{S}=0, k_{D}=1$. Contrary to the white areas, the colored areas indicate self-stabilization condition.

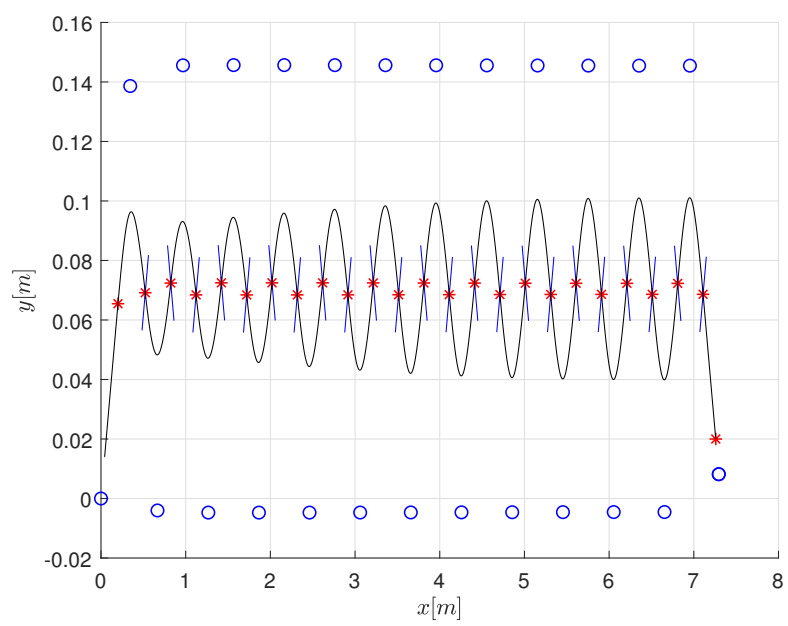

Fig. 8: The projection of the VLIP motion in horizontal plane for 25 steps when $k_{S}=0, k_{D}=0$. The black curves represent the CoM evolution, the blue circles represent the stance foot placements, and the blue lines represent the swiching manifold. 

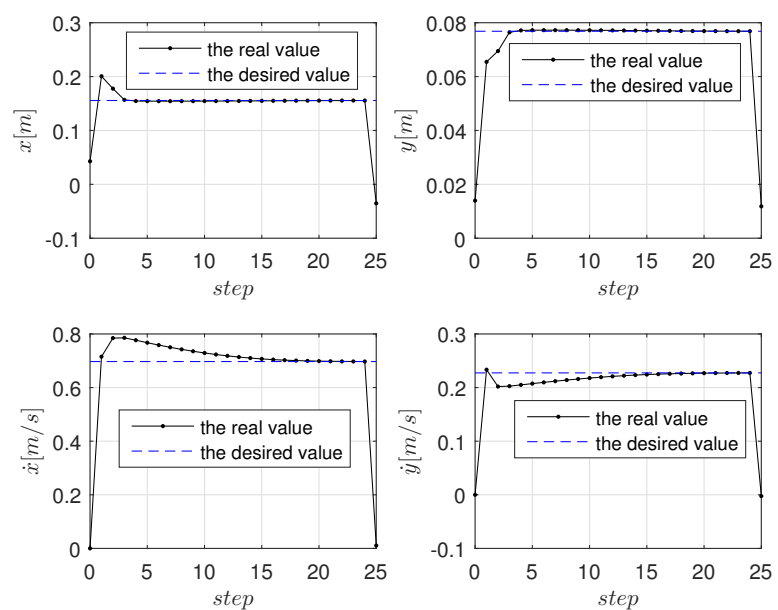

Fig. 9: CoM position and velocity evolutions for 25 steps when $k_{S}=0, k_{D}=0$ in the local reference frame.

As shown in figure 8 , for the case when $k_{S}=0, k_{D}=0$, the CoM of the pendulum starts from a position close to the stance foot with a zero velocity. A change of the landing position of the swing foot along $y$ axis can be observed. This is due to the fact that the landing position strategy consists to nullify the initial CoM position error. From figure 9, it can be seen that after the first step, the system has a state different from that of the periodic motion, converges asymptotically to the periodic motion for the following steps, and stops close to the stance foot with a zero velocity during the last step. For the case when $k_{S}=0, k_{D}=1$, similar results are obtained, as shown in figures 10 and 11 . In this case, except for the first and the last steps which use a different landing strategy, the landing positions of the swing foot stay in a straight line i.e. the step width is constant. Faster convergence can be observed for the case $k_{S}=0, k_{D}=0$, which corresponds to the results shown in figure 5 .

\section{Change of the walking velocity}

Due to the self-stabilization property of the control approach based on virtual constraints, different virtual constraints correspond to different walking velocities. When the humanoid has to adapt its walking velocity, the virtual constraints have to be changed. This can be done for example by inclining forward the torso to increase the walking velocity ${ }^{12}$. In this section we explore the possibility to be able to adapt the walking velocity without recomputing new virtual constraints but only based on a feedback on the current walking speed. Different from the method pro- 


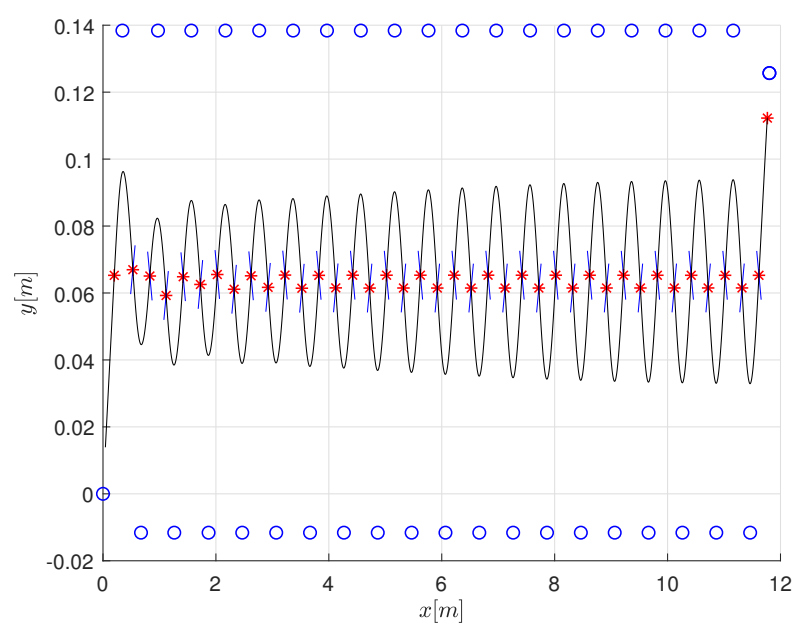

Fig. 10: The projection of the VLIP motion in horizontal plane for 40 steps when $k_{S}=0, k_{D}=1$. The black curves represent the CoM evolution, the blue circles represent the stance foot placements, and the blue lines represent the swiching manifold.

posed by Apostolopoulos et el. in Ref. ${ }^{26}$, the complex procedure of calculating the new periodic motion is unnecessary in this work.

It has been proven in Ref. ${ }^{8}$ that the feedback of the CoM velocity along sagittal axis can provide self-stabilization for a LIP model. How this feedback affects the stability of a VLIP model is discussed here. By introducing the feedback of the walking speed, a new switching manifold was proposed:

$$
\mathbf{S}_{\mathbf{v}}=\left\{(X, Y) \mid\left(X-X^{*-}-l\right)+C\left(Y-Y^{*-}\right)=0\right\} .
$$

As shown in figure 12, the new switching manifold $\mathbf{S}_{\mathbf{v}}$ is a surface with an offset $l$ from the switching manifold proposed in Section 3.1 along $x$ axis. The offset is defined as $l=k_{v}\left(\dot{X}^{*+}-\dot{X}_{k}^{+}\right)$, where $\dot{X}_{k}^{+}$is the CoM velocity along $x$ axis at the beginning of step $k$. It allows to adjust the position of the switching manifold according to the velocity feedback of the CoM for each step. $k_{v}$ is a parameter that must be chosen carefully to satisfy the stability condition.

\subsection{Influence of $C$ and $k_{v}$ on the stability}

Here, $T=0.6 \mathrm{~s}$ is taken and eigenvalues are calculated as functions of $C$ and $k_{v}$ for $\dot{z}_{m}^{d}=0 \mathrm{~m} / \mathrm{s}$ and $\dot{z}_{m}^{d}=-0.1 \mathrm{~m} / \mathrm{s}$. The results of two cases $k_{S}=0, k_{D}=0$ and $k_{S}=0, k_{D}=1$ are shown in figures 13 and 14 respectively.

It can be seen from figures $13 \mathrm{a}$ and $14 \mathrm{a}$ that for a LIP model (corresponding to 

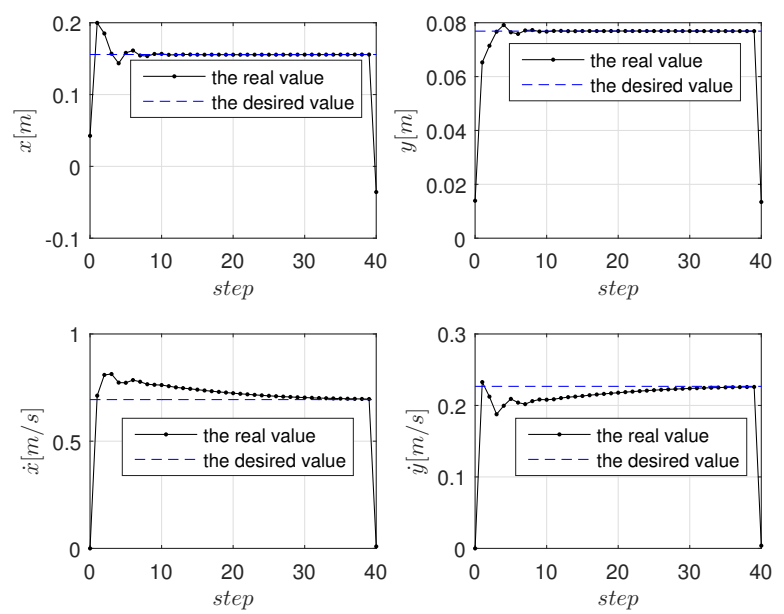

Fig. 11: CoM position and velocity evolutions for 40 steps when $k_{S}=0, k_{D}=1$ in the local reference frame.

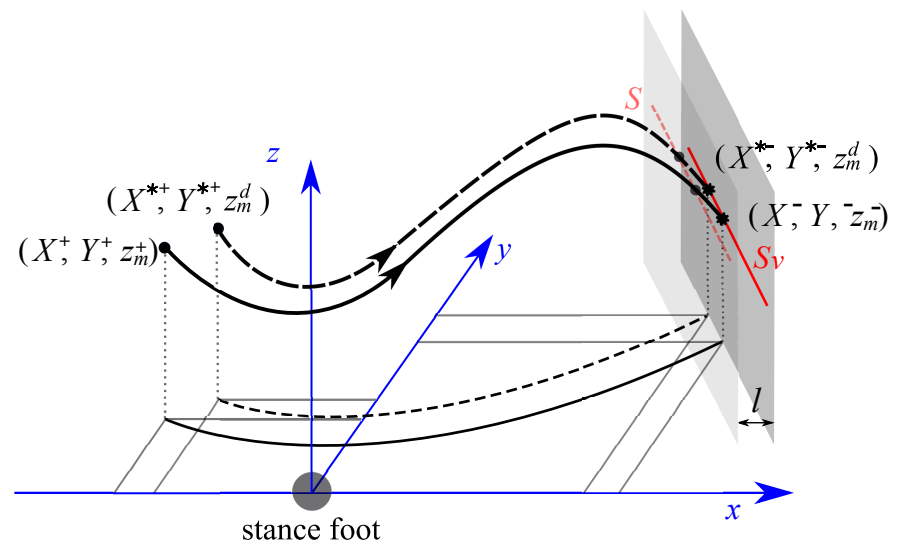

Fig. 12: The step finishes when the CoM crosses the switching manifold, which is represented by the darker gray surface. The curved dashed line is the periodic motion, and the curved solid line is the CoM motion under an initial position perturbation.

the case $\dot{z}_{m}^{d}=0 \mathrm{~m} / \mathrm{s}$ ), stability is obtained only for positive value of $k_{v}$. Contrary to this, it can been seen from figures $13 \mathrm{~b}$ and $14 \mathrm{~b}$ that when $k_{v}=0$, the eigenvalues are smaller than one, which means that the vertical oscillation of the CoM is enough 


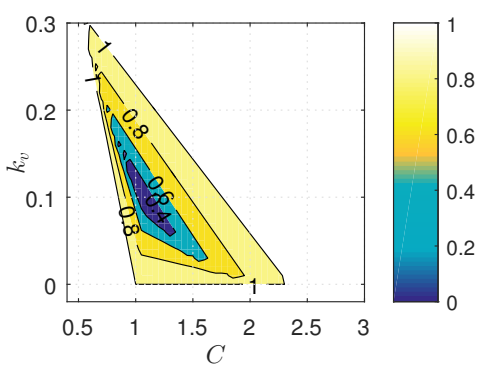

(a) $\dot{z}_{m}^{d}=0 \mathrm{~m} / \mathrm{s}$

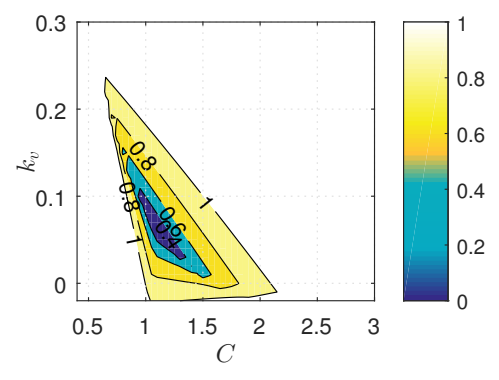

(b) $\dot{z}_{m}^{d}=-0.1 \mathrm{~m} / \mathrm{s}$

Fig. 13: Maximal norm of eigenvalues for different $\dot{z}_{m}^{d}$ as a function of $C$ and $k_{v}$ when $k_{S}=0, k_{D}=0$.

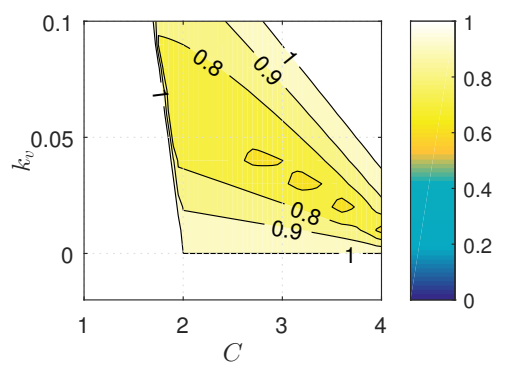

(a) $\dot{z}_{m}^{d}=0 \mathrm{~m} / \mathrm{s}$

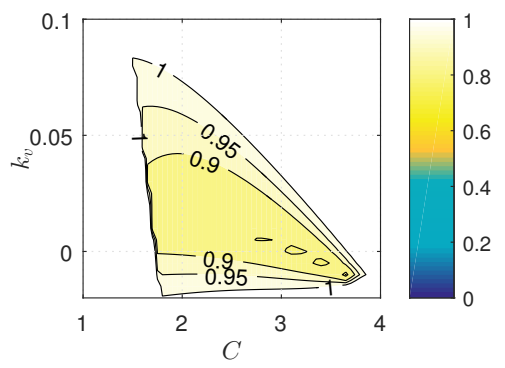

(b) $\dot{z}_{m}^{d}=-0.1 \mathrm{~m} / \mathrm{s}$

Fig. 14: Maximal norm of eigenvalues for different $\dot{z}_{m}^{d}$ as a function of $C$ and $k_{v}$ when $k_{S}=0, k_{D}=1$.

to guarantee convergence to a periodic gait. From figure $13 \mathrm{~b}$, it can be seen that a positive value of $k_{v}$ helps to decrease the amplitude of the eigenvalue when $k_{S}=$ $0, k_{D}=0$, while the effect is not so obvious when $k_{S}=0, k_{D}=1$ as shown in figure $14 \mathrm{~b}$. However, for both cases, a feedback on the CoM velocity to define the switching manifold is useful if a specific walking velocity is desired.

\subsection{Simulations}

This section illustrates the change of switching manifold to control the walking velocity of the robot along the axis $x$. The same virtual constraints are used for all the simulations with desired velocities $\dot{X}^{*+}$ and $\dot{X}^{*+}+\Delta \dot{X}$.

Since the periodic motions for different velocities correspond to different $X^{*-}$ and $Y^{*-}$, these values corresponding to $\dot{X}^{*+}+\Delta \dot{X}$ are unknown. Thus the use 
of the switching manifold (24) does not allow to reach the desired velocity. In order to compensate the static error, an integration term of the velocity error is added into the offset $l$, which gives $l=k_{v}\left(\dot{X}^{*+}-\dot{X}_{k}^{+}\right)+k_{I} \sum_{i=1}^{k}\left(\dot{X}_{i}^{*+}-\dot{X}_{i}^{+}\right)$. The introduction of $k_{v}$ and $k_{I}$ allows the CoM to converge to a new periodic motion without defining explicitly the new periodic gait. While the desired CoM velocity along $x$ axis increases (or decreases), its velocity along $y$ axis will decrease (or increase) correspondingly.

The same parameters as in Section 5.3 are taken: $S=0.3 \mathrm{~m}, D=0.15 \mathrm{~m}, T=$ $0.6 \mathrm{~s}, z_{m}^{d}=0.7 \mathrm{~m}$ and $\dot{z}_{m}^{d}=-0.1 \mathrm{~m} / \mathrm{s}$. The values of $k_{v}$ and $C$ are chosen to be $k_{v}=0.08, C=1.1$ for the case $k_{S}=0, k_{D}=0$ and $k_{v}=0.03, C=2.5$ for the case $k_{S}=0, k_{D}=1$ respectively based the results from figures $13 \mathrm{~b}$ and $14 \mathrm{~b}$. At the $10^{t h}$ step, the desired CoM velocity along $x$ axis $\dot{x}_{m}^{*+}$ is increased by $0.2 \mathrm{~m} / \mathrm{s}$.

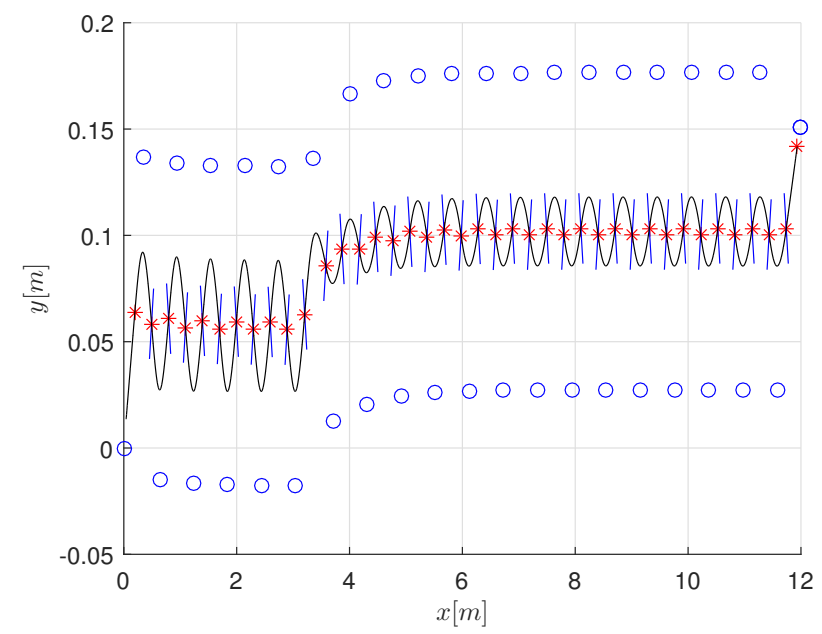

Fig. 15: The projection of the VLIP motion in horizontal plane for 40 steps when $k_{S}=0, k_{D}=0$. The black curves represent the CoM evolution, the blue circles represent the stance foot placements, and the blue lines represent the swiching manifold.

It can be seen from figures 15 and 17 that along with the increase of walking speed, the amplitude of the lateral CoM oscillations is reduced. For the case $k_{S}=0$, $k_{D}=0$ (shown in figure 15), a large change of position along $y$ axis can be also observed, while for the case $k_{S}=0, k_{D}=1$ (shown in figure 17), the robot keeps walking along a straight line despite of the change of speed. From figures 16 and 18, it can be observed that due to the change of the desired velocity from the $10^{\text {th }}$ step, 

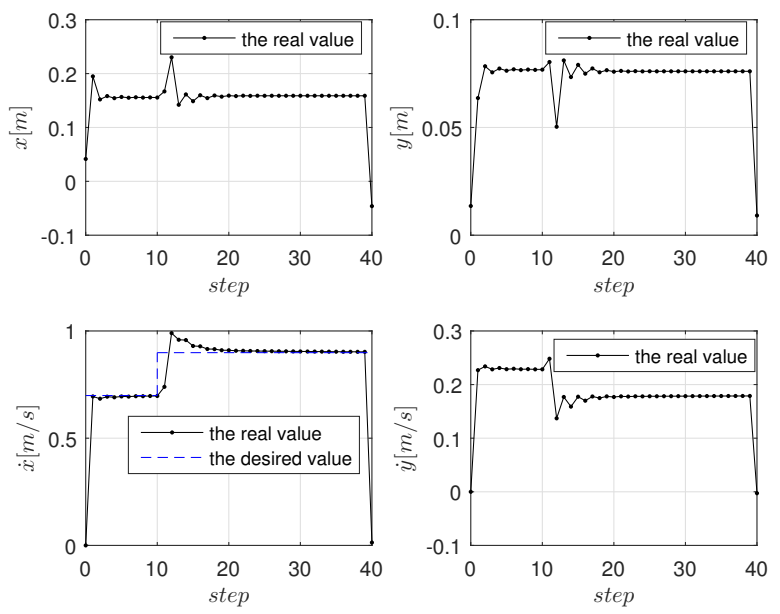

Fig. 16: CoM position and velocity evolutions for 40 steps when $k_{S}=0, k_{D}=0$ in the local reference frame.

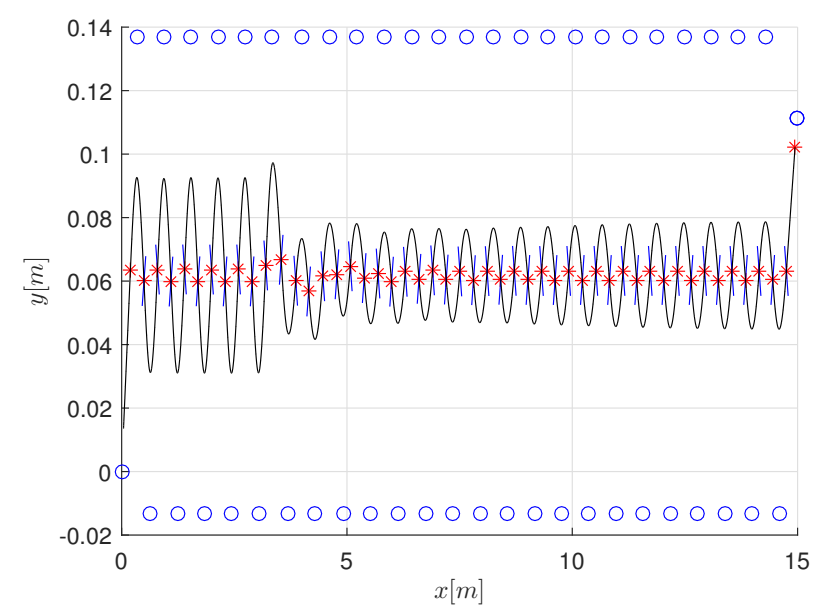

Fig. 17: The projection of the VLIP motion in horizontal plane for 50 steps when $k_{S}=0, k_{D}=1$. The black curves represent the CoM evolution, the blue circles represent the stance foot placements, and the blue lines represent the swiching manifold. 

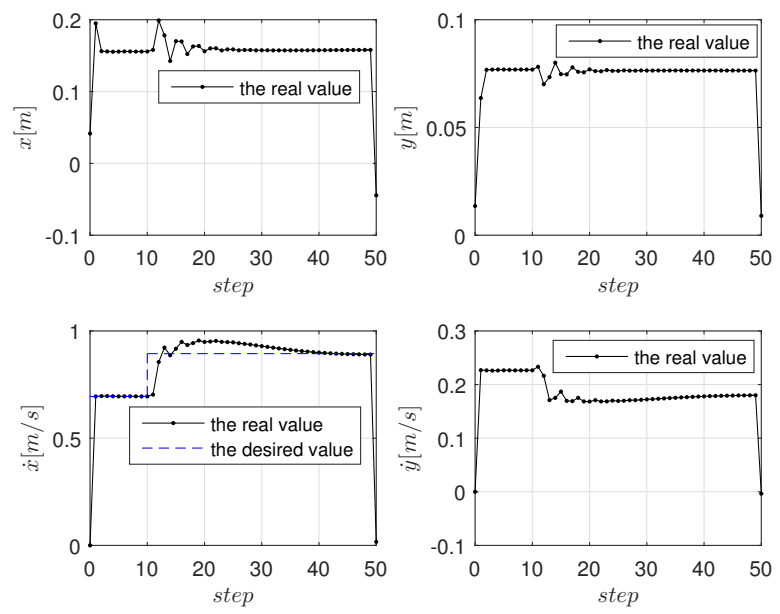

Fig. 18: CoM position and velocity evolutions for 50 steps when $k_{S}=0, k_{D}=1$ in the local reference frame.

the CoM velocity along $x$ axis of the robot is drifted from its original velocity, and eventually converges to the desired velocity. Faster convergence can be observed for the case $k_{S}=0, k_{D}=0$, which corresponds to the results shown in figures 13 and 14 .

\section{Conclusion}

In this paper, the model of the robot is simplified as a VLIP model in order to describe a walking with a limited number of parameters and to highlight the parameters contributing to the stability of walking. The control law is based on virtual constraints. A switching manifold that decides when to switch the stance foot has been proposed, whose position can be adapted to implicitly instead of explicitly change the step timing under disturbances. When the switching manifold is a linear combination of the CoM position in horizontal plane, self-stabilization can be obtained when the vertical velocity of the CoM is negative, i.e. pointing downward. With proper choice of the values of the switching manifold parameters, the walk will asymptotically converge to the periodic motion under disturbance, thus selfstabilization is obtained. The key parameters are : the vertical velocity of the CoM at the transition between steps that must be negative; the position of the foot at the transition that must reduce the position error of the $\mathrm{CoM}$ in the horizontal plane for the next step at least in saggital plane; the orientation of the switching manifold that defines the position of the CoM in horizontal plane at transition between steps. Meanwhile, this paper provided a simple way to define the trajectories 
of the controlled variables, such as the vertical CoM oscillation and the swing foot motion, by proposing a phasing variable that contains the free variables $X$ and $Y$. Moreover, when a PI feedback of the CoM velocity along sagittal axis is taken into account, the walking speed of the robot can converge to another periodic motion with a difference walking speed. This provides a novel approach to find a periodic motion of bipedal robots without using an explicit method.

\section{Acknowledgements}

This work was supported by the China Scholarship Council and national "Robotex" project.

\section{References}

1. Shuuji Kajita, Fumio Kanehiro, Kenji Kaneko, Kazuhito Yokoi, and Hirohisa Hirukawa. The 3d linear inverted pendulum mode: A simple modeling for a biped walking pattern generation. In Intelligent Robots and Systems, 2001. Proceedings. 2001 IEEE/RSJ International Conference on, volume 1, pages 239-246. IEEE, 2001.

2. Cynthia R. Lee and Claire T. Farley. Determinants of the center of mass trajectory in human walking and running. The Journal of experimental biology, 201:2935-2944, 1998.

3. Chris Hayot, Sophie Sakka, Vincent Fohanno, and Patrick Lacouture. Biomechanical modeling of the $3 \mathrm{~d}$ center of mass trajectory during walking. Movement 83 Sport Sciences-Science 83 Motricité, (90):99-109, 2015.

4. Sahab. Omran, Sophie. Sakka, and Yannick Aoustin. Effects of com vertical oscillation on joint torques during $3 \mathrm{~d}$ walking of humanoid robots. Int. J. of Humanoid Robotics, 13(4):1650019, 2016.

5. Gianluca Garofalo, Christian Ott, and Alin Albu-Schäffer. Walking control of fully actuated robots based on the bipedal slip model. In 2012 IEEE International Conference on Robotics and Automation, pages 1456-1463. IEEE, 2012.

6. Kensuke Harada, Kanako Miura, Mitsuharu Morisawa, Kenji Kaneko, Shin'ichiro Nakaoka, Fumio Kanehiro, Tokuo Tsuji, and Shuuji Kajita. Toward human-like walking pattern generator. In 2009 IEEE/RSJ International Conference on Intelligent Robots and Systems, pages 1071-1077. IEEE, 2009.

7. Christine Chevallereau and Yannick Aoustin. Self-stabilization of 3d walking via vertical oscillations of the hip. In Robotics and Automation (ICRA), 2015 IEEE International Conference on, pages 5088-5093. IEEE, 2015.

8. Qiuyue Luo, Victor De-León-Gómez, Anne Kalouguine, Christine Chevallereau, and Yannick Aoustin. Self-synchronization and self-stabilization of walking gaits modeled by the three-dimensional lip model. IEEE Robotics and Automation Letters, 3(4):3332-3339, 2018.

9. Miomir Vukobratović and Branislav Borovac. Zero-moment point-thirty five years of its life. International journal of humanoid robotics, 1(01):157-173, 2004. 
10. Santiago Martinez, Juan Garcia-Haro, Juan Victores, Alberto Jardon, and Carlos Balaguer. Experimental robot model adjustments based on force-torque sensor information. Sensors, 18(3):836, 2018.

11. Dalila Djoudi and Christine Chevallereau. Stability analysis of a walk of a biped with control of the zmp. In 2005 IEEE/RSJ International Conference on Intelligent Robots and Systems, pages 2461-2467. IEEE, 2005.

12. Eric R Westervelt, Christine Chevallereau, Jun Ho Choi, Benjamin Morris, and Jessy W Grizzle. Feedback control of dynamic bipedal robot locomotion. CRC press, 2007.

13. Hassan K Khalil. Nonlinear systems. Upper Saddle River, 2002.

14. Pierre-Brice Wieber. Trajectory free linear model predictive control for stable walking in the presence of strong perturbations. In Humanoid Robots, 2006 6th IEEE-RAS International Conference on, pages 137-142. IEEE, 2006.

15. S. Kajita, F. Kanehiro, K. Kaneko, K. Fujiwara, K. Harada, K. Yokoi, and H. Hirukawa. Biped walking pattern generation by using preview control of zero-moment point. In 2003 IEEE International Conference on Robotics and Automation (Cat. No.03CH37422), volume 2, pages 1620-1626 vol.2, Sept 2003.

16. Toru Takenaka, Takashi Matsumoto, and Takahide Yoshiike. Real time motion generation and control for biped robot-1 st report: Walking gait pattern generation. In Intelligent Robots and Systems, 2009. IROS 2009. IEEE/RSJ International Conference on, pages 1084-1091. IEEE, 2009.

17. Michael A Hopkins, Dennis W Hong, and Alexander Leonessa. Dynamic walking on uneven terrain using the time-varying divergent component of motion. International Journal of Humanoid Robotics, 12(03):1550027, 2015.

18. Johannes Englsberger, Christian Ott, Máximo A Roa, Alin Albu-Schäffer, and Gerhard Hirzinger. Bipedal walking control based on capture point dynamics. In Intelligent Robots and Systems (IROS), 2011 IEEE/RSJ International Conference on, pages 4420-4427. IEEE, 2011.

19. Johannes Englsberger, Christian Ott, and Alin Albu-Schäffer. Threedimensional bipedal walking control based on divergent component of motion. IEEE Transactions on Robotics, 31(2):355-368, 2015.

20. Juan Alejandro Castano, Zhibin Li, Chengxu Zhou, Nikos Tsagarakis, and Darwin Caldwell. Dynamic and reactive walking for humanoid robots based on foot placement control. International Journal of Humanoid Robotics, 13(02):1550041, 2016.

21. Majid Khadiv, Alexander Herzog, S Ali A Moosavian, and Ludovic Righetti. Step timing adjustment: A step toward generating robust gaits. In Humanoid Robots (Humanoids), 2016 IEEE-RAS 16th International Conference on, pages 35-42. IEEE, 2016.

22. Víctor De-León-Gómez, Qiuyue Luo, Anne Kalouguine, J Alfonso Pámanes, Yannick Aoustin, and Christine Chevallereau. An essential model for generating walking motions for humanoid robots. Robotics and Autonomous Systems, 112:229-243, 2019. 
23. Brent Griffin and Jessy Grizzle. Nonholonomic virtual constraints for dynamic walking. In Decision and Control (CDC), 2015 IEEE 54th Annual Conference on, pages 4053-4060. IEEE, 2015.

24. Shuuji Kajita, Hirohisa Hirukawa, Kensuke Harada, and Kazuhito Yokoi. Introduction to humanoid robotics, volume 101. Springer, 2014.

25. Sylvain Miossec and Yannick Aoustin. A simplified stability study for a biped walk with underactuated and overactuated phases. The International Journal of Robotics Research, 24(7):537-551, 2005.

26. Sotiris Apostolopoulos, Marion Leibold, and Martin Buss. Transitioning between underactuated periodic orbits: An optimal control approach for settling time reduction. International Journal of Humanoid Robotics, 15(06):1850027, 2018 .

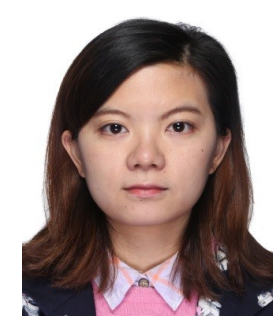

Qiuyue Luo received her M.S. degree from Northwestern Polytechnical University (China) in 2016. She is currently pursuing her Ph.D. in Robotics at Laboratoire des Sciences du Numérique de Nantes (LS2N) in Centrale Nantes (France). Her research interest is bipedal walking.

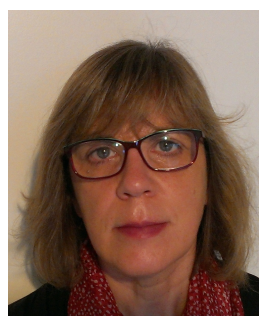

Christine Chevallereau graduated from Ecole Nationale Supérieure de Mécanique, Nantes, France in 1985, and received the Ph.D. degree in Control and Robotics from Ecole Nationale Supérieure de Mécanique, Nantes in 1988. Since 1989, she has been with the CNRS in the Institut de Recherche en Communications et Cybernétique de Nantes and then Laboratoire des Sciences du Numérique de Nantes. She is deputy director of the LS2N.

Christine Chevallereau is the author of over 100 technical publications or communications. Her research interests include modeling and control of manipulators and locomotor robots, in particular biped, and bio-inspired robots.

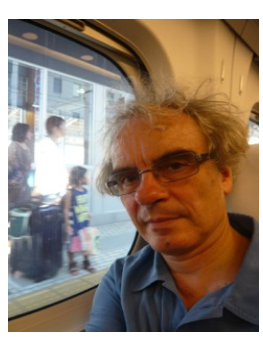

Yannick Aooustin received his M.S. degree in 1984 Engineering from the University of Nantes, France, and his Ph.D. degree in Automatic Control from the University of Nantes, Country, in 1987. He obtained his research degree for leading research and PhD students in 2006. From 1990 to 2015, he was at the University of Name, as Associate Professor. $\mathrm{He}$ is currently an Full Professor in the Department of Physics, the University of Nantes,

Yannick Aoustin is the author of over 50 technical publications. His research interests include mechanical systems under actuated legged robots, bipedal, nonlin- 
ear observers, and bio-mechanics. He is also a topic editor of "International Journal of Advanced Robotic Systems" and member of the editorial board of the journal "Multibody system dynamics". 